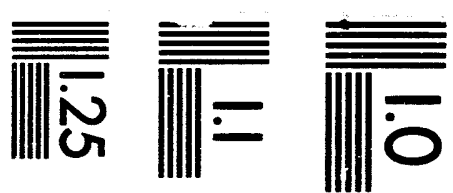

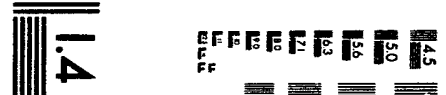

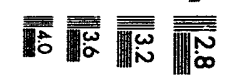

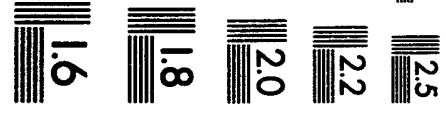



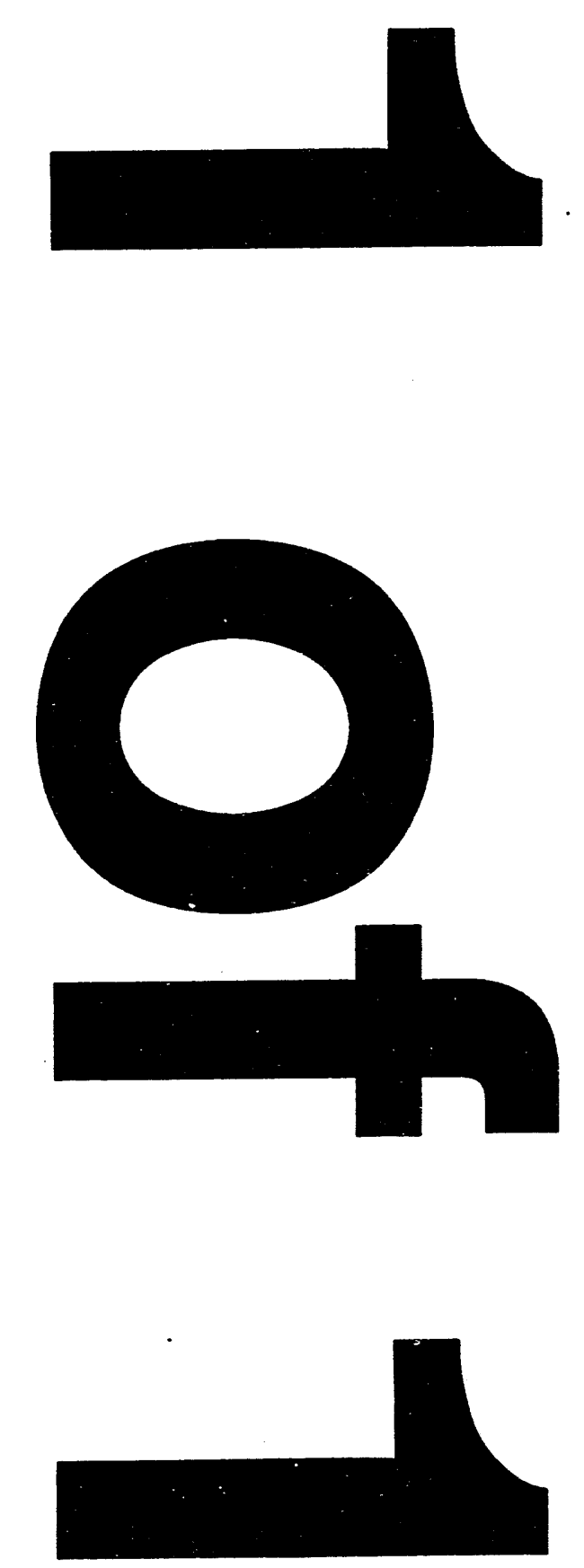


$$
\text { DOE|EK| } 13949 \ldots 5
$$

\section{Molecular Aspects of Transport in Thin Films of Controlled Architecture}

Technical Summary

July 1,1992 - June 30, 1993
Hom

DEC 301993

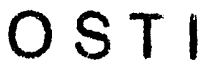

Work on this project has progressed in two principal areas during the past year: (1) characterization of diffusion in swollen polymer films both with and without a barrier layer, and (2) initial investigations of the molecular aspects of swelling using enhanced Raman spectroscopy. Progress in each area will be described separately, although of course there is extensive crossfertilization among the students working in each area.

\section{Transport Phenomena in Swollen Thin Films}

In the previous budget period we had developed a detailed transport model appropriate to the use of optical waveguides to characterize the permeation of probe molecules into swollen polymer films. In particular we concentrated on the permeation of fluorescein into $\mathrm{H}_{2} \mathrm{O}$-swollen poly(2vinylpyridine). Initial experiments relied on fiber-optic collection and transfer to a spectrometer. However, in the past year we have investigated a system in which the fiber-collection is replaced by direct imaging with a charge-coupled device (CCD) array camera. Belo'w we describe the improvements in performance and the subsequent new information about transport which were obtained.

Reprise of the Model. The equation used to fit the data obtained from the permeation experiments is derived from a consideration of the fluorescence from a waveguide. Equation 1 describes the fluorescence from a thin film, where

$I(t)=\int_{0}^{1} F(t) I_{0} e^{-F(t) x \varepsilon} d x$

$I(t)$ is the fluorescence as a function of time, $F(t)$ is the fluorophore concentration in the film as a function of time, $I_{0}$ is a constant which includes terms for the various instrument-dependent parameters, $x$ is the position along the guide, 1 is the length of the guide imaged by the collectiun optics, and $\varepsilon$ is the absorptivity of the fluorophore. It is necessary to derive an expression for the fluorophore concentration in the film. We begin with Fick's Second Law, where $F(z, t)$ is the

$\frac{\partial F(z, t)}{\partial t}=D \frac{\partial^{2} F(z, t)}{\partial z^{2}}$

concentration in the film as a function of time and position, in the thickness direction, in the film, $t$ is the time, $\mathrm{D}$ is the diffusion coefficient, and $\mathrm{z}$ is the position in the film in the thickness direction. In order to solve this equation, an appropriate set of boundary conditions are:

$$
\begin{aligned}
& F=F_{0}, z>w, t \geq 0 \\
& F=0,-w<z<w, t=0 \\
& \partial F / \partial z=0, z=0, t \geq 0
\end{aligned}
$$

These boundary conditions yield an expression for the concentration of fluorophore in the film as a function of position in the thickness direction and time, as shown in equation 7 , 
$F(t)=\kappa C_{0}\left(1-\frac{8}{\pi^{2}} \sum_{n=0}^{\infty}\left[\frac{(-1)^{n}}{(2 n+1)^{2}} \exp \left[\frac{-t D \pi^{2}(2 n+1)^{2}}{4 w^{2}}\right] \sin \left\{\frac{(2 n+1) \pi}{2}\right\}\right]\right)$

where $F_{0}$ is the bulk solution concentration of fluorophore, $w$ is the film thickness, $n$ is an integer, $\kappa$ is the partition coefficient across the film-solution boundary, and all of the other constants are as previously defined. This equation can be used to relate the film response to the transport properties only if the various constants are first determined. The five unknowns are $w$, the swollen film thickness, $\varepsilon$, the molar absorptivity, $\kappa$, the partition coefficient, $\mathrm{I}_{0}$, and $\mathrm{D}$. The swollen film thickness can be determined from a measurement of the mode distribution, and $\varepsilon$ can be combined with $\kappa$, resulting in a new parameter $\xi$. Therefore, a three parameter fit is performed to obtain $\xi, I_{0}$, and $D$. $D$ can then be used to examine the effect of changes in film structure on permeation. A typical fit along with a plot of the residuals is shown in Figure 1.

Experimental Effects and Results, Several artifacts in the acquisition of data with the fibercollection scheme were identified and rectified with CCD-collection. All of them were ultimately traced to movement of the spatial position corresponding to maximum intensity. Although there was no way in which this could have been identified in the fiber-collected data, Figure 2 readily demonstrates that CCD data collection allows one to identify and circumvent such artifacts. Taken together the collection of curves shown in Figure 2 indicates pathological behavior in this particular experiment, since the maximum intensity (related to saturation of the film) is seen to vary depending on the pixel observed. In addition, in some of the data the intensity actlially goes through a maximum and begins to decrease again.

The first facet of the permeation of fluorescein into P2VP films to be examined was the effect of the bulk solution concentration on the calculated diffusion coefficients. Data for a variety of conditions taken with both collection schemes is sunmarized in Table 1. It is immediately obvious that the sample-to-sample variability is much larger than the error associated with the determination of $D$ in a given sample. This effect can most likely be attributed to the difficulty in fabricating polymer films with reproducible microstructure. It is known that the rate of permeation in the film is greatly affected by the supermolecular architecture of the film, which, in turn, is determined, at least partially, by its thermal history. In the present experiments, the samples were removed from the vacuum oven at $120^{\circ} \mathrm{C}$ and placed in ambient conditions and allowed to cool. Samples generally took less than 5 minutes to reach room temperature. Since the samples were rapidly cooled to room temperature from above their $T_{g}$, they are expected to have a more open structure relative to slowly cooled samples. The effect of the rate of cooling through the glass transition temperature on the rate of diffusion has been previously examined by Thomas and Windle ${ }^{1}$ who found that the rate of diffusion increases with the rate of cooling. In order to test this hypothesis, several samples were cooled slowly in the vacuum oven and the permeation experiment was performed with a bulk solution concentration of $100 \mathrm{nM}$. These experiments resulted in diffusion coefficients of $2.51 \times 10^{-12} \pm 1.4$ $\times 10^{-15} \mathrm{~cm}^{2} / \mathrm{s}$ and $5.01 \times 10^{-12} \pm 3.6 \times 10^{-15} \mathrm{~cm}^{2} / \mathrm{s}$, or an average $3.76 \times 10^{-12} \pm 2.7 \times 10^{-15} \mathrm{~cm}^{2} / \mathrm{s}$, as compared to an average of $7.22 \times 10^{-12} \pm 8.7 \times 10^{-13} \mathrm{~cm}^{2} / \mathrm{s}$ for rapidly cooled samples.

Table 1 also clearly demonstrates the effect of the data collection scheme on the results. The fiber optic cannot be brought sufficiently close to the coupling point to include the peak intensity in the region it images, leading to a systematic error in the determined value of $D$. Since the peak interisity can be observed in the CCD collection scheme, these effects can be avoided. The use of the $C C D$ for data collection also improves the reproducibility in each concentration. The only concentration for which this can be directly compared is $100 \mathrm{nM}$, where three experiments have been performed with each collection scheme. The fiber optic collection yields a $40.8 \%$ RSD in D for 100 $\mathrm{nM}$, while the CCD collection yields a $10.1 \% \mathrm{RSD}$ in D for $100 \mathrm{nM}$. Since the 1/e propagation distance of the guided radiation is approximately $15 \mathrm{~mm}$, or 100 pixels and decreases as the fluorophore concentration in the film increases, and the fiber position is ca. $5 \mathrm{~mm}$, or 30 pixels, from

${ }^{1}$ Thomas, N. L.; Windle, A. H. Polymer 1980, 21, 613. 
the peak position and the fiber positioning is only reproducible to ca. $3 \mathrm{~mm}$, or 20 pixels, the fiber's imaged region is centered $53 \mathrm{~mm}$, or $30-50$ pixels, from the peak. A shift of this magnitude, $33 \%$ the 1/e propagation distance, in the best case, would be expected to seriously degrade the reproducibility of the data. The effect of bulk solution concentration is also depicted in Table 1 . The CCD data demonstrates that the fluorescein mass transport coefficient exhibits no systematic concentration dependence in the range $1 \mathrm{nM} \leq$ [fluorescein] $\leq 10 \mu \mathrm{M}$. The fiber optic data show a slight increase with increasing concentration, however, as demonstrated above, these data are likely to be in error.

It is also useful to compare the results obtained with the optical waveguide experiment to results from other systems. Smith has reported a diffusion coefficient of $1.6 \times 10^{-13} \mathrm{~cm}^{2} / \mathrm{s}$ for 7(diethylamino)-4-nitrobenz-2-oxa-1,3-diazole into a $1 \mu \mathrm{m}$ thick poly(methylmethacrylate) film containing $18 \%$ chlorobenzene, using fluorescence redistribution after pattern photobleaching ${ }^{2}$. Smith's slower diffusion coefficient, as compared with those reported here, can be attributed to the differences in the permeant, the polymer film and the lower liquid content of the film. Wang and $\mathrm{Xia}^{3}$ have used a similar technique to examine the diffusion of trans- and cis-azobenzene in poly(vinyl acrylate) containing $4.1 \%$ toluene. They have found a diffusion coefficient of $8.1 \times 10^{-13}$ $\mathrm{cm}^{2} / \mathrm{s}$ for the trans form and $1.1 \times 10^{-12} \mathrm{~cm}^{2} / \mathrm{s}$ for the cis form. Again the slower diffusion can be attributed to the fact that the film contains less solvent than the samples in this study. Nevertheless, these results indicate that the values of $D$ are comparable to those typically obtained for solventswollen films.

Thus, the optical waveguide methodology has proven effective in characterizing Fickian diffusion, just as for the work in Case II systems completed earlier. With these techniques in hand for characterizing the phenomenological parameters associated with diffusion in thin $\mu \mathrm{m}$-scale films, it seems appropriate to focus the remainder of our attention on characterizing the molecular scale motions responsible for transport in polymer films.

\section{Investigations of Swelling on the Molecular Length Scale}

We had proposed to use the spatial localization of the electric field in thin $\mathrm{Ag}$ or Au layers interspersed at different points within a polymer to study the changes in chain structure associated with solvent swelling in Case II diffusion processes. Raman scattering from the molecular segments in close proximity $(\leq 100 \AA)$ to the metal particles will be used to characterize segments of the film undergoing swelling. In last year's annual report we detailed the progress on instrumentation that was needed to extract information concerning the molecular scale motions of interest using Au-enhanced near infrared surface Raman scattering.

Surface enhanced Raman scattering (SERS)-active metal island films placed within $\mu \mathrm{m}$-sized polymer sandwich thin film structures were explored as molecular-level probes of Case II diffusion events for $n$-hexane swelling of poly(styrene). There are three major results which have come to light in the past year. (1) A comparison of $\mathrm{Ag}$ and $\mathrm{Au}$ films indicates that strong SERS signals are obtained from $\mathrm{Au}$ island structures interspersed to various depths within poly(styrene), while simultaneously avoiding photo-oxidation problems typically encountered with Ag-enhancing structures. (2) The physical spread of the Au island structure in the depth direction was determined to be $\leq 200 \AA$ by Auger depth profiling and physical profilometry. Combining this physical spread and the SERS-distance dependence $d(1 / e) \approx 50 \AA$ yields a spatial resolution for this technique $\leq 300$ $\AA$. (3) Large changes in the vibrational spectrum with solvent influx were noted for the poly(styrene) features at $1070 \mathrm{~cm}^{-1}$ and $1371 \mathrm{~cm}^{-1}$. In particular, changes in the latter band were interpreted in te $\mathrm{ms}$ of an increase in chain mobility, with solvent passage, which allows the pendant phenyl moieties

\footnotetext{
$2 \varsigma$ nith, B. A. Macromolecules 1982, 15, 469.

${ }^{3}$ Wang, C. H.; Xia, J. L. J. Phys. Chem. 1992, 96, 190.
} 
to align parallel to the semimajor axes of the oblate spheroidal particles, thus increasing their effective scattering cross-section.

Ag ys. Au Enhancing Structures. Poly(styrene) SERS spectra obtained using Ag and $\mathrm{Au}$ enhancing structures are shown in Figure 3. Broad features, generated by graphite-like photodegradation products, dominate the Ag SERS spectrum in the region of interest between 1100 and $1650 \mathrm{~cm}^{-1}$, even when spectra were acquired at excitation irradiances below $10 \mathrm{~mW} / \mathrm{cm}^{2}$. None of these features are visible in the Au SERS spectrum. Even after prolonged exposure to nearinfrared excitation radiation Au-enhanced SERS spectra show none of the features associated with sample degradation characteristic of the Ag-enhanced spectra. Thus, for all subsequent work Au enhancing structures were used exclusively. In general the spectra from bulk and Au SERS samples are quite similar in the 900 to $1600 \mathrm{~cm}^{-1}$ region, there are, however, some minor differences. The relative intensity of the $v_{9 b}$ (Wilson mode) in-plane ring vibration at $1592 \mathrm{~cm}^{-1}$ in the SERS sample is smaller than the same mode in the bulk sample (at $1602 \mathrm{~cm}^{-1}$ ). In addition, a new mode at 1560 $\mathrm{cm}^{-1}$ was always present in poly(styrene) SERS spectra, and two modes at 1223 and $1275 \mathrm{~cm}^{-1}$ were occasionally observed. Infrared bands assigned to a complex skeletal mode, including $\delta(\mathrm{CH})$ previously observed at 1222 and $1275 \mathrm{~cm}^{-1}$ for $\alpha$-, and B-syndiotactic poly(styrenes) respectively ${ }^{4}$, may correspond to the modes observed here.

Spatial Resolution. A number of experiments were performed to determine the spatial resolution of the Raman signal collected from a typical sandwich structure consisting of polymermetal island film-polymer films. The resolution should be a function of three factors: (1) Au SERS enhancement factors, i.e., the relative magnitudes of the signal originating adjacent to the metal islands and that originating from the bulk polymer, (2) the spatial distribution of the metal islands in the direction of permeant motion, and (3) the depth of the polymer overlayer in the probe region.

(1) A simple set of composite sample structures was fabricated in order to ascertain the physical location from which the majority of the signal was arising. These demonstrated that the signal is dominated by the region containing the Au metal island structure. The lack of signal from the polymer-only region gives conclusive proof that the spectroscopic signals are arising from the vicinity ( $\leq 100 \AA$ ) of the metal island structures. (2) AES depth profiling was performed to determine the distribution of the metal islands in the permeation direction. Intensities of the Auger features at $45 \mathrm{eV}$ (for $\mathrm{Au}$ ) and at $240 \mathrm{eV}$ (for C) from a sample consisting \& a polymer film coated with a $40 \AA$ mass thickness $\mathrm{Au}$ island film were used to generate atom composition percentages. Typical results for a sample with the Au structure at the surface are shown in Figure 4. It is clear that the transition from Au-containing region to pure poly(styrene) takes less than $200 \AA$. In addition, expected artifacts, such as the knock-on effect and other factors contributing to lack of resolution in the depth direction, would cause the observed width of the Au islands to be larger, not smaller, meaning that the number recovered in these experiments is an upper limit on the physical thickness of the enhancing structure. Therefore, based on this information, it is clear that the width of the distribution of metal islands atop the polymer film is $\leq 200 \AA$. This result is fortuitous, since there is no a priori reason to expect the depth distribution to be especially narrow, and the narrower the depth distribution of the islands, the more localized in space the spectroscopic signals become. The sampling region can also be estimated from this result, since we know that the Raman signal falls off to $1 / \mathrm{e}$ of its adsorbate intensity roughly in $50 \AA$ for oblate spheroidal particles, such as those used in these studies 5 . Since, the $50 \AA$ can be on either side of the metal particles, the overall sampling region has a depth $\leq 300 \AA$, i.e., about $3 \%$ of the thickness of a $1 \mu \mathrm{m}$ polymer film. (3) The final parameter which could affect the spatial resolution is lateral thickness uniformity. The region of polymer film sampled, using a line focus produced by a cylindrical lens, was approximately $10 \mu \mathrm{m} \mathrm{x}$ $5 \mathrm{~mm}$. Film thickness was found to vary by less than $4 \%$ over any $5 \mathrm{~mm}$ region parallel to the focus direction. In summary, these results demonstrate the excellent spatial resolution attainable in the

\footnotetext{
${ }^{4}$ (a) Nyquist, R. A.: Putzig, C. L. Leugers, M. A.; McLachlan, R. D.; Thill, B. Appl. Spect., 1992, 46, 981-987. (b) Kobayashi, M.; Nakooki, T.; Isihara, N. Macromolecules 1989, 22, 4377.

5Roth, P. G.; Boerio, F. J. J. Polym. Sci., Polym. Phys. Ed. 1987, 25, 1923.
} 
thickness direction using Au SERS-enhancing structures to probe $\mu \mathrm{m}$-scale polymer thin film structures.

Molecular Reorganization. Macroscopic observations of Case II diffusion in glassy polymers, show that several events might affect the SERS signal of poly(styrene) sandwich structures upon exposure to n-hexane. First, some relaxation of the polymer chains should occur in response to osmotic stress created by the approach of the solvent front. This reorientation is a key part of Case II theory, since it is postulated to be the physical event which controls the velocity of the front. Next, growth of solvent features and further changes in the polymer spectrum might be anticipated as the front passes over the probe region and polymer chains are partially solvated. Finally, after the front has passed over the metal island film, and the concentration of permeant reaches a constant value, the solvent features would be expected to reach a steady-state signal level.

The time course for spectra of both annealed and unannealed poly(styrene) film structures were obtained. Permeation results for samples prepared with and without annealed poly(styrene) overlayers were very similar both in terms of the types and temporal evolution of spectral changes observed. While solvent features were not visible in the annealed samples, spectra obtained during the permeation of unannealed films clearly showed solvent modes at $870,1080,1125$, and $1457 \mathrm{~cm}^{-1}$, as well as polymer bands at 1230 and $1371 \mathrm{~cm}^{-1}$, which increased dramatically in intensity. The temporal dependence of the $1371 \mathrm{~cm}^{-1}$ polymer band and the $1125 \mathrm{~cm}^{-1}$ solvent band is shown in Figure 5. It is interesting to note that changes in mode intensity begin almost immediately after the solvent is introduced to the sample and continue in a smooth fashion throughout the run and that the approach to steady state is significantly faster for the solvent than for the poly(styrene). Crazing, the formation of microscopic cracks in response to the osmotic stress built up at the solvent front, is likely responsible for delivering some solvent to the vicinity of the enhancing island structures well in advance of the main solvent front, if we interpret the buildup of the $1125 \mathrm{~cm}^{-1}$ band purely in terms of an increased solvent number density. Alternatively, the fact that the data can be fit with an exponential build-up may simply be indicative of the Fickian leading edge in the near surface region for this structure, however the craze-induced solvent delivery is a characteristic feature of the poly(styrene)-n-alkane system which has been observed in the optical waveguide permeation experiments performed in our laboratory.

The behavior of the poly(styrene) band at $1371 \mathrm{~cm}^{-1}$ is more interesting. This is assigned to the $v_{15}$ band of monosubstituted benzenes and corresponds to a $\left(B_{2} u\right)$ aromatic $\mathrm{C}-\mathrm{H}$ in-plane bending, $\delta(\mathrm{CH})$. It is somewhat surprising that a mode which is nominally non-totally symmetric in the native molecule should show so strongly in the SERS spectrum of the solvent swollen film, since it is empirically observed that totally symmetric vibrations typically dominate SERS spectra. The key feature of its behavior Luring permeation is that it reaches steady-state more slowly than the solvent band, indicating that polymer relaxation continues to occur after the solvent signal has saturated. In addition, since the polymer number density near the metal islands likely does not change significantly during the course of the permeation, it is probable that the changes in the poly(styrene) band are caused by reorientation of the side chains to bring the phenyl rings into more favorable orientation for interaction with the electronic resonances of the oblate particles. These changes can be interpreted in terms of polymer reorganization necessary to accommodate the osmotic stress associated with passage of the solvent front.

\section{Plans for the Coming Year}

As we saw earlier the optical waveguide methodology has proven effective in characterizing both Fickian and Case II diffusion from a phenomenological perspective. With these techniques in hand for characterizing the phenomenological parameters, they can be regarded as routine tools for characterizing new thin film polymer/solvent systems. Thus, we do not plan any additional development work on these experimental capabilities. Instead, now that we have demonstrated the utility of the SERS-structures for examination of transport in thin polymer films, we will concentrate all of our attention on understanding the molecular aspects of transport. In particular, we will exploit 
the localized vibrational spectroscopy capability by turning to a system, poly(methylmethacrylate) $\mathrm{CH}_{3} \mathrm{CN}$, in which mechanical crazing does not cause such difficulties as those encountered in the poly(styrene)/n- $\mathrm{C}_{6} \mathrm{H}_{14}$ system studied to-date. Acetonitrile is chosen as solvent due to its strong and readily separable vibrational marker associated with the C-N stretching near $2300 \mathrm{~cm}^{-1}$. Previous work on the poly(styrene) samples with deuterated hexane $\left(n-C_{6} D_{14}\right)$ indicated that, although the $C$ D stretch could be observed, it was sufficiently weak so as to not be usable as a marker for studying the dynamics. We will examine both thin ( $\mu \mathrm{m}$-scale) and ultrathin ( $\mathrm{t} \sim 1-2$ molecules) film samples using both bulk liquid (for thin films) and vapor phase (for ultrathin film) transport to deliver solvent. The purpose of examining two systems simultaneously is to try to decouple the solvent transport from the molecular reorganization, in order to better understand the interplay of the two. It is particuiarly critical to decouple the two in light of the observation in the poly(styrene) system that solvent signal is saturated long before changes in the polymer reorganization are complete.

In addition to switching to a new experimental system, we will investigate the effect of polymer processing parameters on the physico-chemical behavior observed. Investigations of Fickian diffusion in P2VP and of Case II transport in poly(styrene) point individually to the importance of polymer thin film preparation conditions on obtaining reproducible experimental results. Therefore, we will concentrate heavily on careful characterization of the thermal history of the polymer films as an experimental parameter to be understood. We have recently automated the vacuum-baking apparatus used for annealing polymer film samples, and we will exploit this capability to control the time which the sample is held above $T_{g}$ and the rate of cooling back to 300 $\mathrm{K}$.

In addition to continuing the work already begun on the vibrational spectroscopy of these systems we will concentrate some effort on partially aligned composite systems consisting of carrier molecules interspersed in a polymer matrix. Over the past several years we have had an industrially sponsored program with the goal of understanding how to construct oriented arrays of metalloproteins, by using genetic engineering techniques to introduce unique reactive sites on the protein exterior. Since the same genetic engineering techniques can be used to design the binding characteristics of the ligand binding site in molecular-carrier proteins, these systems may be of some significant interest as components of composite film permselective membrane systems. In particular, the degree of control which can be exerted over the binding characteristics is impressive. For example, it has been demonstrated in myoglobin that the ratio of binding constants of $\mathrm{CO}$ and $\mathrm{O}_{2}$ can be varied by a factor of 100 simply by changing the geometric and electrostatic characteristics of the binding site. Thus, we anticipate the impact of these systems by devoting a portion of our work to the fabrication and characterization of composite polymer/metalloprotein structures. The key questions to be addressed initially are: (1) stability, and (2) retention of function. Of course, these two questions are not completely separate. Initially we will examine systems composed of genetically engineered myoglobin introduced into films of bovine serum albumin. V/e will address the stability issue by examining the resonance Raman spectroscopy of the heme prosthetic group. Marker bands which report on core size, oxidation state, and spin state are readily available in the $1300-1600 \mathrm{~cm}^{-1}$ region. In addition, we will examine the capability of the metalloprotein to retain its binding function by examining the capability to bind $\mathrm{O}_{2}$ as a function of processing parameters.

\section{Publications Acknowledging DOE Support}

N.F. Fell, Jr. and P.W. Bohn, "Mass Transport Phenomena in Thin Films of Poly(2-vinylpyridine) Studied via Optical Guided Wave Techniques," Analyt. Chem., submitted.

P.A. Drake and P.W. Bohn, "Localized Raman Scattering Probes of Molecular Scale Motions in Case II Swelling of Poly(styrene) in n-Hexane," Analyt. Chem., submitted. 
H.-G. Hong, M. Jiang, S.G. Sligar, and P.W. Bohn, "Cysteine-Specific Surface Tethering of Genetivally Engineered Cytochromes for Fabrication of Metalloprotein Nancstructures," Langmuir, submitted.

P.W. Bohn, "Aspects of Structure and Energy Transport in Artificial Molecular Assemblies," Ann. Rev. Phys. Chem., in press.

H.-G. Hong, P.W. Bohn, and S.G. Sligar, "Optical Determination of Surface Density in Oriented Metalloprotein Nanostructures," Analyt. Chem. 65, 1635-1638 (1993).

P.W. Bohn and N.F. Fell, "Species Transport and Distribution in Thin Polymer Films Using Eigenmode Distributions in Integrated Optical Structures," in Polymer Solutions and Interfaces, D. Rubingh and I. Noda, Eds., pp. 359-384, Pergamon, New York (1992). 


\begin{tabular}{|c|c|c|c|c|}
\hline $\begin{array}{c}\text { Data Acquisition } \\
\text { Technique }\end{array}$ & $\begin{array}{c}\text { Fluorescein Bulk } \\
\text { Solution } \\
\text { Concentration }\end{array}$ & $\begin{array}{c}\text { Average Mass } \\
\text { Transport } \\
\text { Coefficient, D } \\
\left(\mathrm{cm}^{2} / \mathrm{s}\right)\end{array}$ & $\begin{array}{c}\text { Standard } \\
\text { Deviation of } \\
\text { Average D }\left(\mathrm{cm}^{2} / \mathrm{s}\right)\end{array}$ & $\begin{array}{c}\text { Average Fit Error } \\
\text { (Number of } \\
\text { Replicates) } \\
\left(\mathrm{cm}^{2} / \mathrm{s}\right)\end{array}$ \\
\hline \multirow{2}{*}{ Fiber Optic } & $100 \mathrm{nM}$ & $2.82 \times 10^{-11}$ & $1.15 \times 10^{-11}$ & $4.04 \times 10^{-13}(3)$ \\
& $10 \mathrm{nM}$ & $1.40 \times 10^{-11}$ & $5.71 \times 10^{-12}$ & $1.41 \times 10^{-14}(2)$ \\
& $1 \mathrm{nM}$ & $8.95 \times 10^{-12}$ & - & $1.5 \times 10^{-13}(1)$ \\
\hline \multirow{2}{*}{ CCD } & $10 \mu \mathrm{M}$ & $1.39 \times 10^{-12}$ & - & $4.02 \times 10^{-14}(1)$ \\
& $1 \mu \mathrm{M}$ & $6.90 \times 10^{-12}$ & - & $5.34 \times 10^{-14}(1)$ \\
& $500 \mathrm{nM}$ & $5.05 \times 10^{-12}$ & $1.36 \times 10^{-12}$ & $2.50 \times 10^{-14}(4)$ \\
& $100 \mathrm{nM}$ & $7.22 \times 10^{-12}$ & $7.27 \times 10^{-13}$ & $8.64 \times 10^{-13}(3)$ \\
& $50 \mathrm{nM}$ & $4.38 \times 10^{-12}$ & $5.16 \times 10^{-13}$ & $9.08 \times 10^{-15}(2)$ \\
& $1 \mathrm{nM}$ & $4.00 \times 10^{-12}$ & - & $9.92 \times 10^{-16}(1)$ \\
\hline
\end{tabular}

Table 1. Results for mass transport coefficient determinations as a function of data acquisition technique and bulk fluorescein solution concentration. The standard deviation of the average $D$ is a measure of the sample-to-sample reproducibility, while the average fit error is an indication of the error associated with the determination of $D$ for each sample. The average fit error was calculated from the square root of the average of the squares of the individual fit errors. 


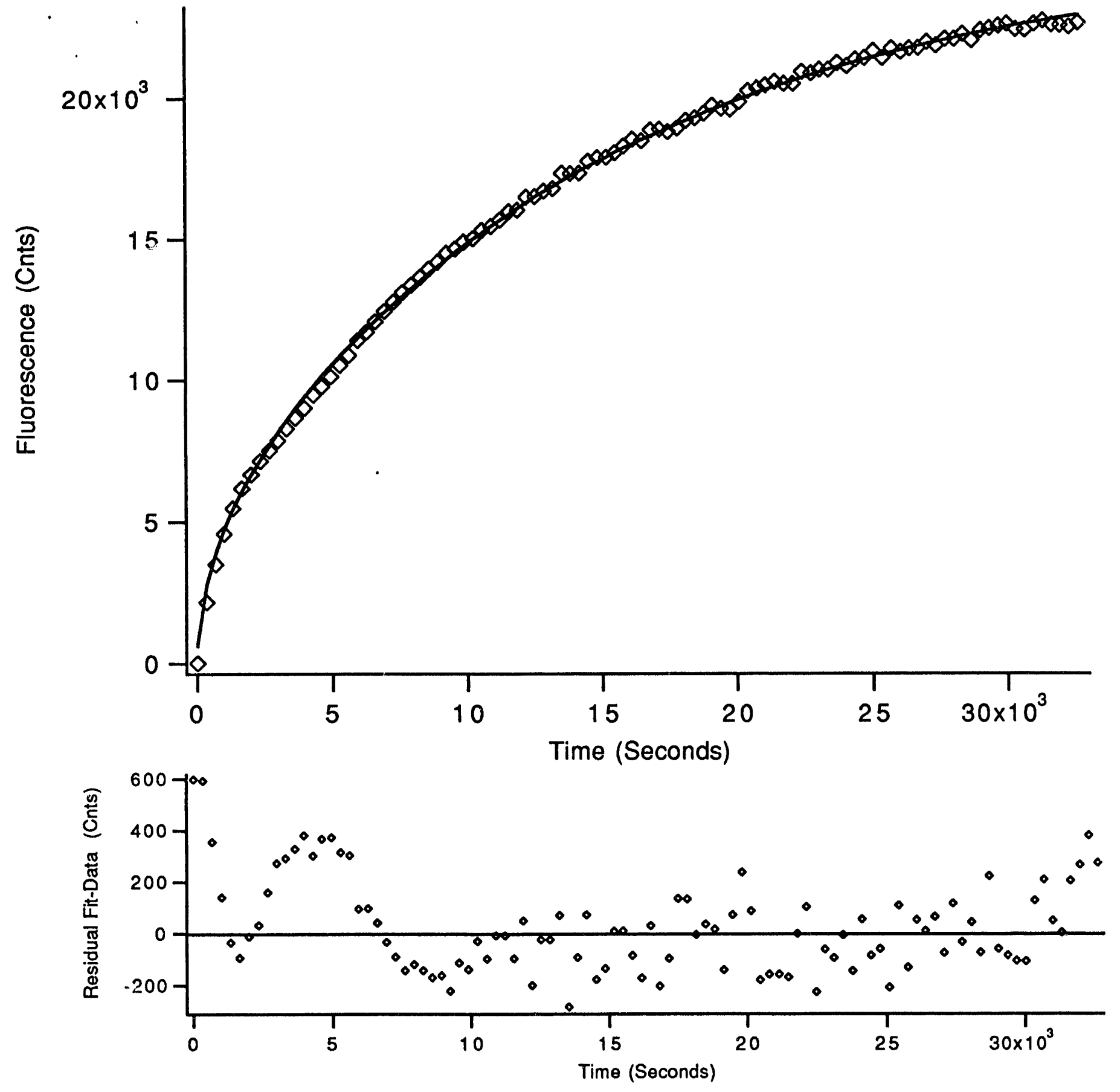

Figure 1: A representative plot of (top) the fit (line) and experimental data (points) and (bottom) the residual between the experimental data and the calculated fit. Note that the residual is ca. two orders of magnitude smaller than the values of the data points at long time. This data set is from an uncoated P2VP film exposed to $1 \mu \mathrm{M}$ fluorescein with data collected via the CCD camera. 


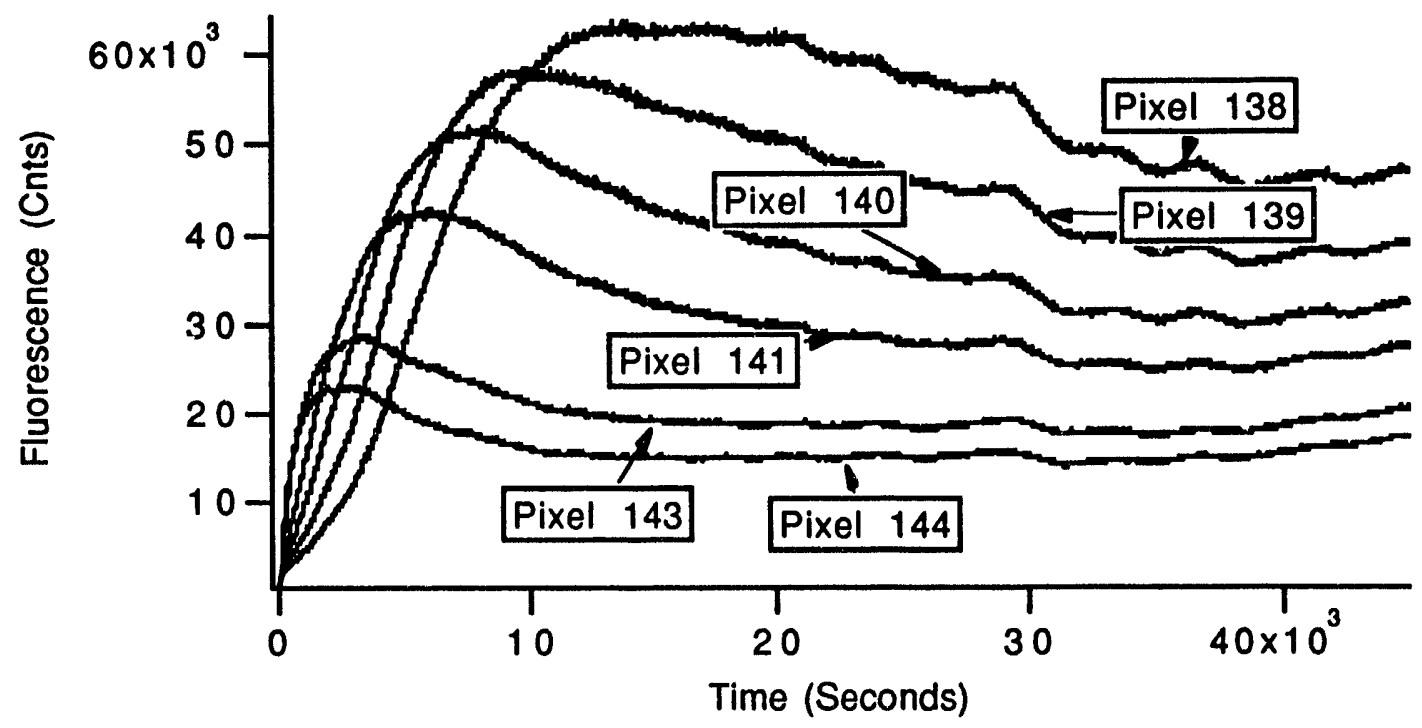

Figure 2: The effect of measurement position along the propagation direction on the response curves, showing the high solution concentration $(1 \mu \mathrm{M})$ tumover effect in the response curves caused by the excitation intensity decay rate overtaking the increase in concentration. The entry point to the sample region of the cell occurs at pixel 132. In the earlier pixels (132-137) there is anomalous behavior due to the signal levels exceeding the linear range of the CCD. 


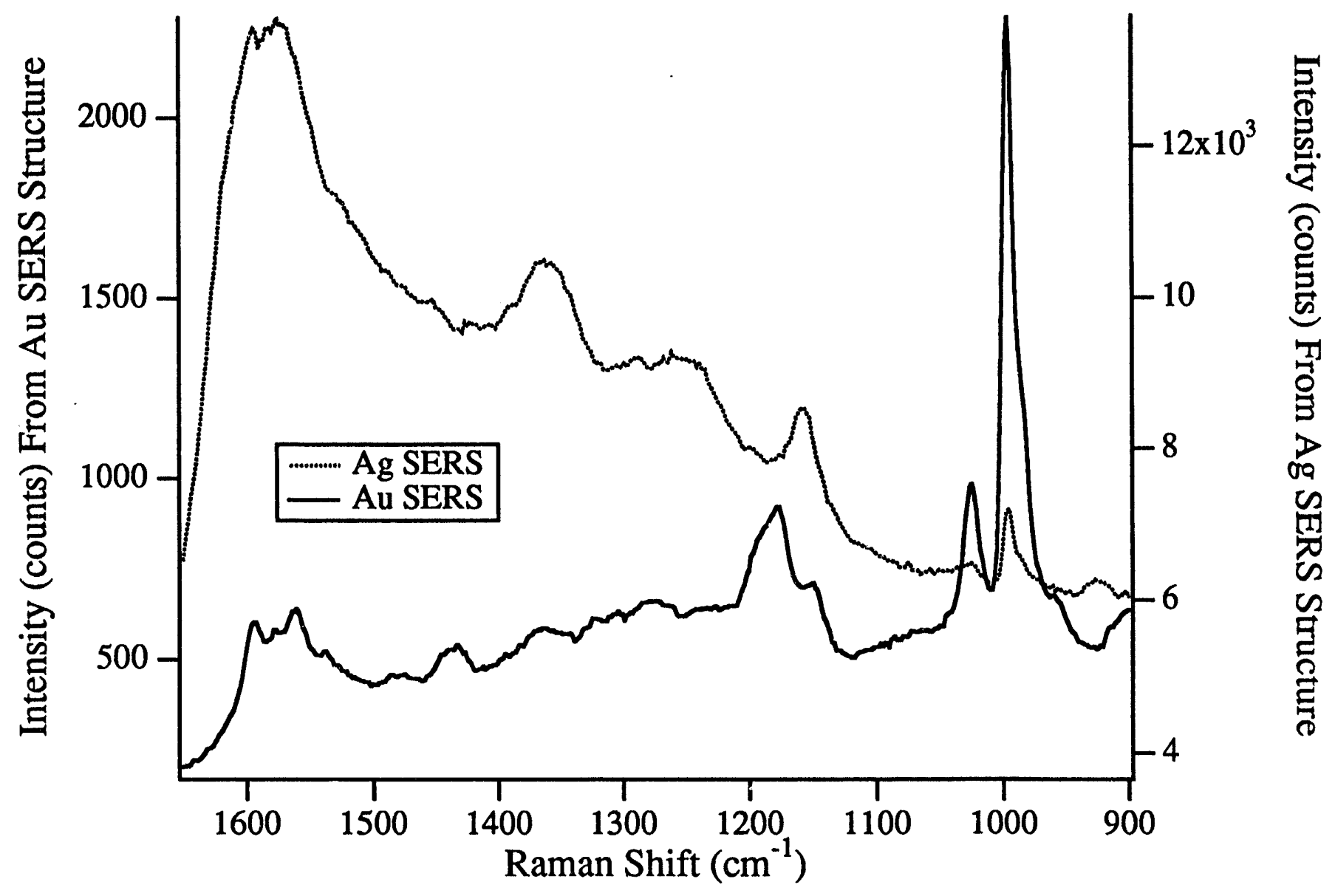

Figure 3: Spectra obtained for sandwich structures of metal (Ag or Au) islands with poly(styrene). The Ag spectrum was excited at $488.0 \mathrm{~nm}$, while the Au spectrum was excited at $750.0 \mathrm{~nm}$. Noti the difference in the $y$-axis scales for the two enhancing media. 


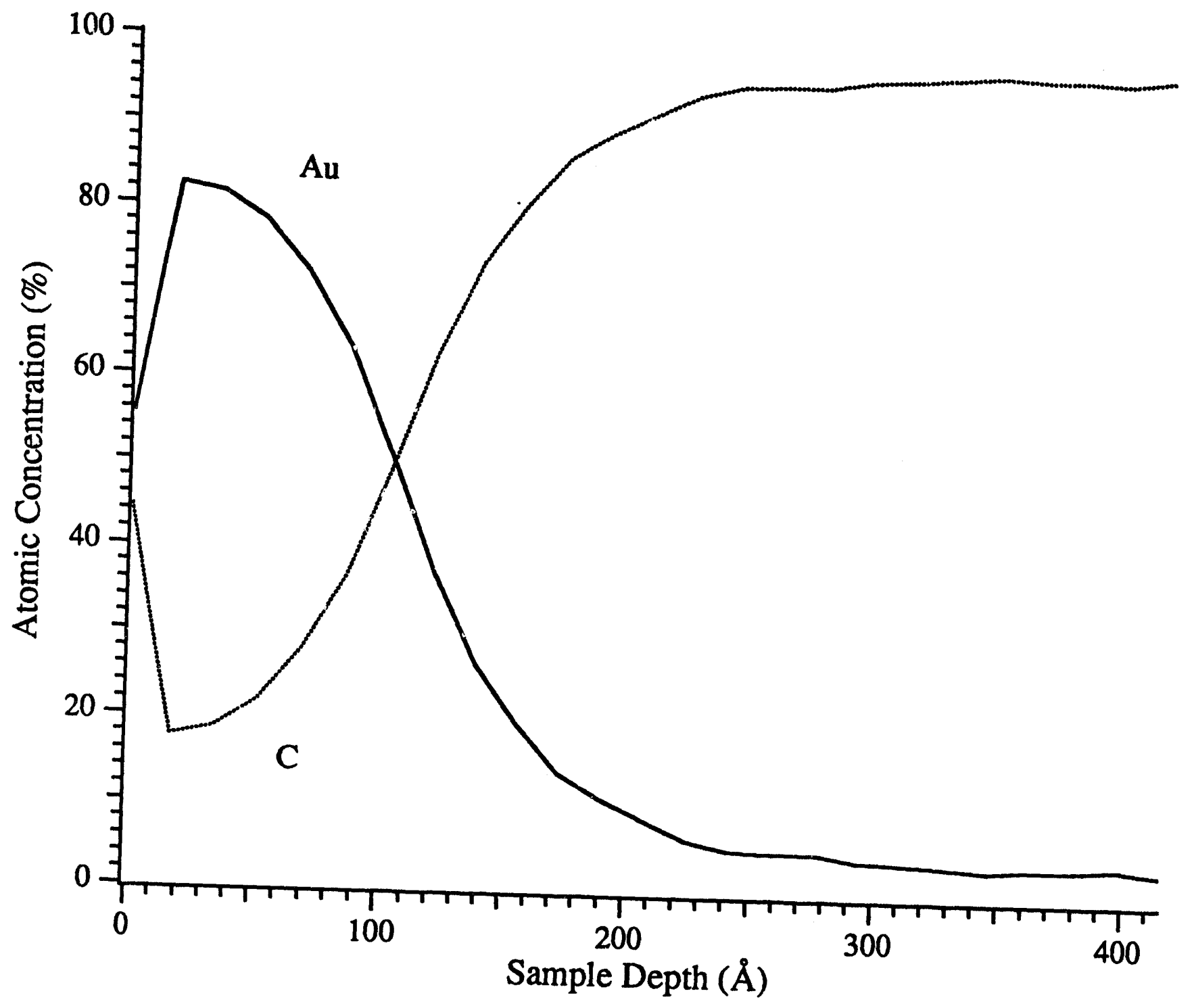

Figure 4: Composition vs. depth of a sample which originally contained a $40 \AA$ mass thickness Au film
confined to the surface. 


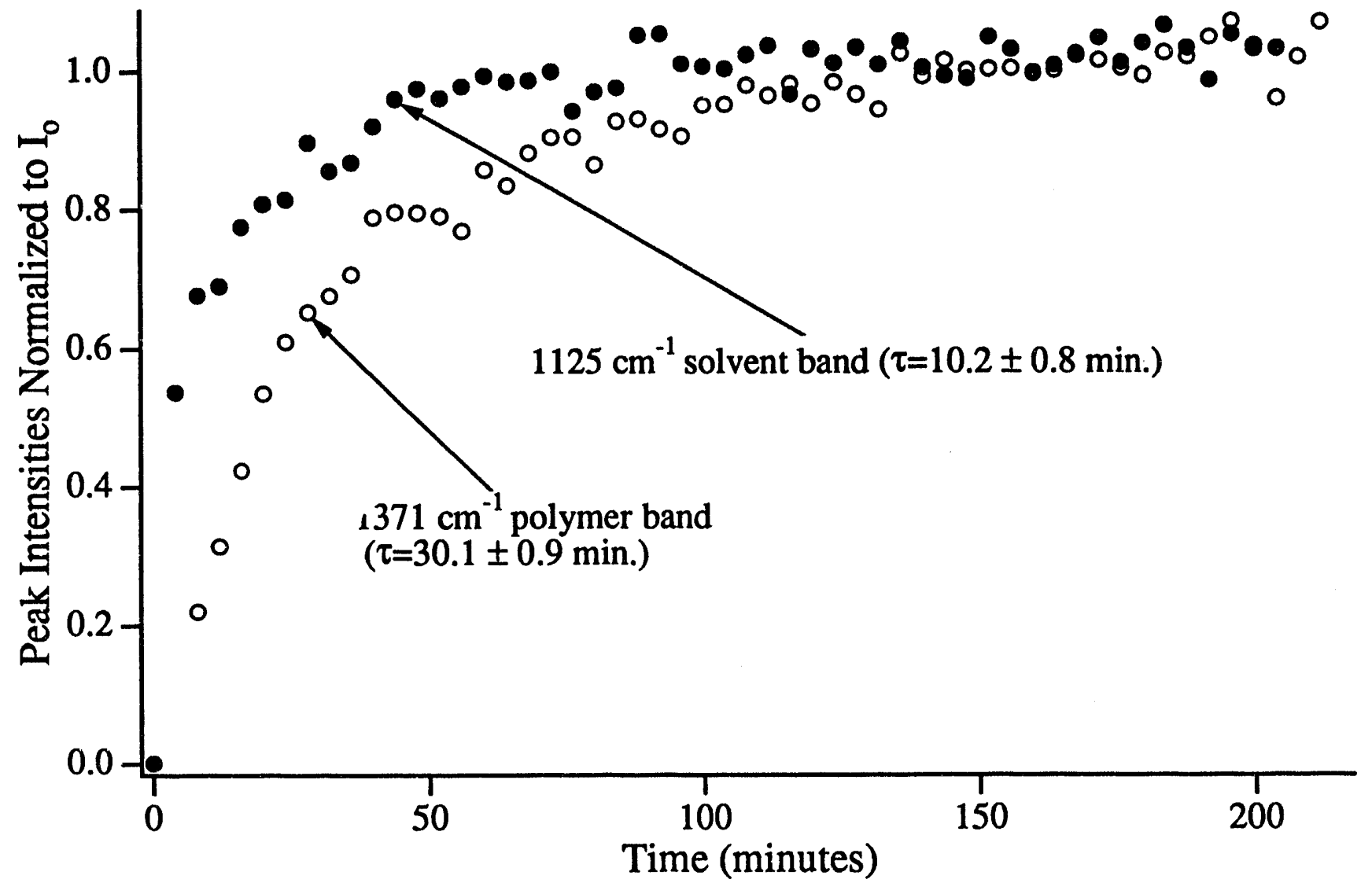

Figure 5: Temporal development of the Raman bands at $1125 \mathrm{~cm}^{-1}$, due to $n-\mathrm{C}_{6} \mathrm{H}_{14}$, and $1371 \mathrm{~cm}^{-1}$, which is assigned to an in-plane ring deformation of the phenyl sidechain in poly(styrene). Fits to the empirical model, $I=I 0(1-e-t / t)$, were used to derive values for $t$ shown in the inset.

\section{DISCLAIMER}

This report was prepared as an account of work sponsored by an agency of the United States Government. Neither the United States Government nor any agency thereof, nor any of their employees, makes any warranty, express or implied, or assumes any legal liability or responsibility for the accuracy, completeness, or usefulness of any information, apparatus, product, or process disclosed, or represents that its use would not infringe privately owned rights. Reference herein to any specific commercial product, process, or service by trade name, trademark, manufacturer, or otherwise does not necessarily constitute or imply its endorsement, recommanufacturer, or otherwise does not necessarily constitute or States Government or any agency thereof. The views and opinions of authors expressed herein do not necessarily state or reflect those of the United States Government or any agency thereof. 


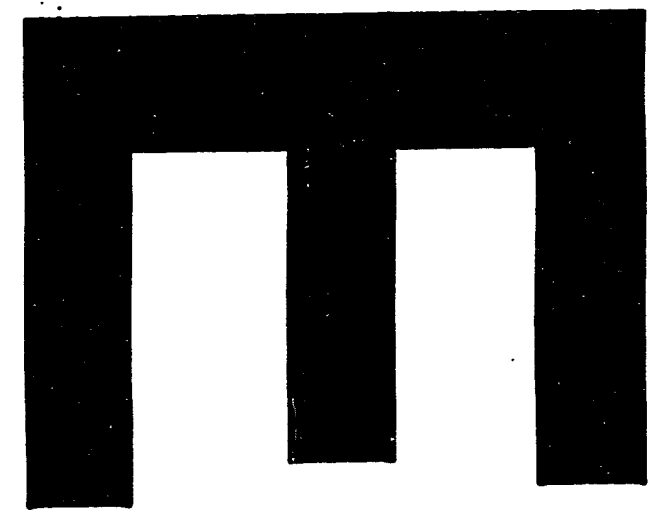

9
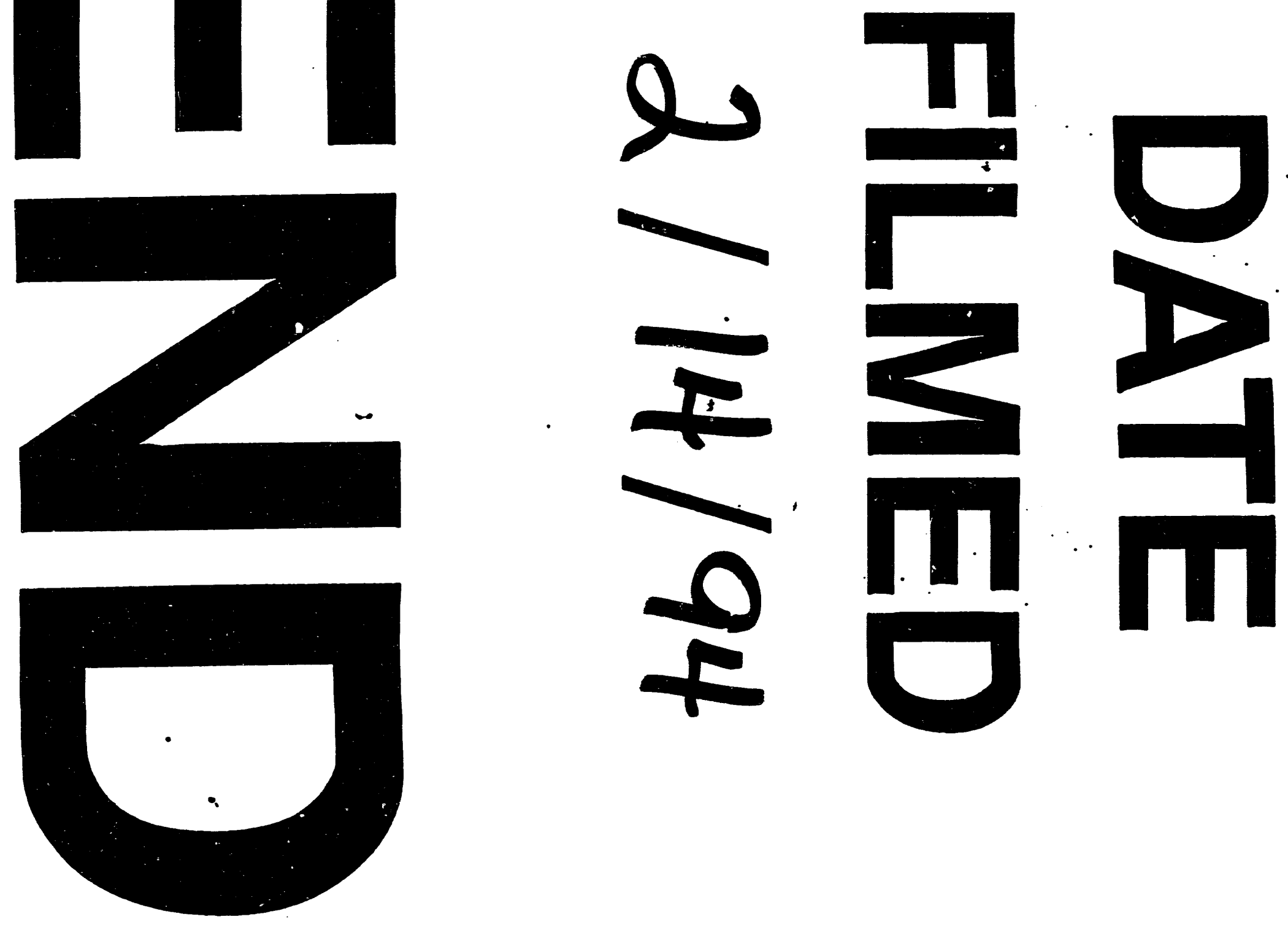
(

.

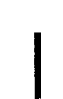

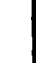

